\title{
Pacific
}

Journal of

Mathematics

\section{CONTROLLING TIETZE-URYSOHN EXTENSIONS}

MARC FRANTZ 


\title{
CONTROLLING TIETZE-URYSOHN EXTENSIONS
}

\author{
MARC FRANTZ
}

\begin{abstract}
This paper explores some of the possibilities for controlling properties of continuous extensions of continuous functions. For example, particular attention is paid to the problem of preserving some desirable common property (e.g. pairwise disjointness, partition of unity, etc.) of a collection of functions, when the entire collection is extended simultaneously and continuously from a closed subset $A$ of a normal topological space $X$ to the whole space. The functions treated here are mainly real-valued, but in the last section, a procedure introduced by Dugundji is used to show how to preserve the equicontinuity of a collection of functions whose ranges lie in a locally convex metric linear space.
\end{abstract}

0. Introduction. If $A$ is a closed subset of a normal topological space $X$, and if $f: A \rightarrow \mathbf{R}$ is continuous function, then by the extension theorems of Tietze $[\mathbf{T}]$ and Urysohn $[\mathbf{U}]$ (the combination of which we will refer to as [TU]), there exists a continuous extension $\hat{f}$ of $f$ to all of $X$. Several generalizations of these results have been obtained, notably Katětov's result [K] for uniformly continuous functions on uniform spaces, and Dugundji's result [D] for continuous functions into linear topological spaces. In this article, we present some results which deal with the degree of control we have over the extensions of continuous functions; that is, how we may choose the sets where extended functions take on certain specified values, or satisfy some predetermined relationship.

In Section 1, we show that we may choose, with reasonable topological restrictions, any finite number of disjoint closed subsets of $X$ as level sets for $\hat{f}$.

We show in Section 2 that if we have a continuous function $f$ and a nonnegative (or nonpositive) continuous function $g$ defined on a closed subset $A$ of a compact metric space $X$, such that their product $f g$ satisfied $f g=\left.h\right|_{A}$ for some continuous function $h$ : $X \rightarrow \mathbf{R}$, then under suitable conditions $f$ and $g$ may be extended 
to continuous functions $\hat{f}$ and $\hat{g}$ on all of $X$ such that $\hat{f} \hat{g}=h$. At the end of this section we outline a much more general problem, some aspects of which are treated in later sections.

Section 3 contains two propositions on extending pairwise disjoint continuous functions. The second one employs a construction recently used by Mandelkern in [M].

In section 4, we show that a partition of unity defined on a closed subset of a normal space may be extended to the whole space.

In section 5 we show that an extension procedure defined by Dugundji in [D], when applied to functions from a closed subset $A$ of a metric space $(X, d)$ to a locally convex metric linear space $(Y, \varrho)$, preserves the equicontinuity of any pointwise bounded equicontinuous collection of functions. A related result (Corollary 9) states that for any compact subset $\left\{f_{\gamma}\right\}$ of $C(A, Y)$, there exists a compact subset $\left\{\hat{f}_{\gamma}\right\}$ of $C(X, Y)$, such that $\left.\hat{f}_{\gamma}\right|_{A}=f_{\gamma}$ for each $\gamma$.

1. Extending level sets. Let $X$ be a normal topological space, let $A$ be a closed subset of $X$, and let $f: A \rightarrow \mathbf{R}$ be continuous. By [TU], there exists a continuous extension $\hat{f}$ of $f$ to all of $X$. Moreover, if $A_{i}:=f^{-1}\left(\left\{a_{i}\right\}\right)$ for arbitrary real numbers $a_{1}, \ldots, a_{n}$ in the range of $f$, then observe that in extending $f$ we have also extended the level sets $A_{i}$ of $f$ to level sets $\hat{A}_{i}:=\hat{f}^{-1}\left(\left\{a_{i}\right\}\right)$ of $\hat{f}$. It is therefore reasonable to ask whether, starting with subsets $\hat{A}_{i}$ of $X$ satisfying $\hat{A}_{i} \cap A=A_{i}$ for each $i$, we can find a continuous extension $\hat{f}$ of $f$ such that $\hat{f}^{-1}\left(\left\{a_{i}\right\}\right)=\hat{A}_{i}$ for each $i$.

Clearly this implies that each $\hat{A}_{i}$ must be a closed $G_{\delta}$-subset of $X$. Moreover, if we assume that $a_{1}<\cdots<a_{n}$, and let

$$
\begin{aligned}
\hat{U}_{0} & :=\hat{f}^{-1}\left(\left(-\infty, a_{1}\right)\right), \hat{U}_{1}:=\hat{f}^{-1}\left(\left(a_{1}, a_{2}\right)\right), \cdots, \hat{U}_{n-1} \\
& :=\hat{f}^{-1}\left(\left(a_{n-1}, a_{n}\right)\right), \hat{U}_{n}:=\hat{f}^{-1}\left(\left(a_{n}, \infty\right)\right)
\end{aligned}
$$

then obviously the $\hat{U}_{i}$ are pairwise disjoint and $X \backslash\left(\bigcup_{i=1}^{n} \hat{A}_{i}\right)=$ $\bigcup_{i=0}^{n} \hat{U}_{i}$. Notice that, generally speaking, $\hat{A}_{i}$ must separate $X \backslash \hat{A}_{i}$ into two disjoint open sets.

It turns out that if the sets $\hat{A}_{i}$ satisfy these minimal requirements, then the desired extension $\hat{f}$ exists (Theorem 2). The following theorem, used as an auxiliary result for Theorem 2 , is in its own right a reasonable extension of [TU] in the case when $f$ is a bounded function. 
TheOREM 1. Let $X$ be a normal topological space, let $A$ be a closed subset of $X$, and let $f: A \rightarrow[c, d]$ be a continuous function, such that the sets $C:=f^{-1}(\{c\})$ and $D:=f^{-1}(\{d\})$ are nonempty. If $\hat{C}$ and $\hat{D}$ are disjoint closed $G_{\delta}$-sets in $X$ such that $\hat{C} \cap A=C$ and $\hat{D} \cap A=D$, then there exists a continuous extension $\hat{f}: X \rightarrow[c, d]$ of $f$ such that $\hat{C}=\hat{f}^{-1}(\{c\})$ and $\hat{D}=\hat{f}^{-1}(\{d\})$.

Proof. Define the function $f_{1}: A \cup \hat{C} \cup \hat{D} \rightarrow[c, d]$ by

$$
f_{1}(x):= \begin{cases}f(x) & \text { if } x \in A \\ c & \text { if } x \in \hat{C} \\ d & \text { if } x \in \hat{D}\end{cases}
$$

Clearly $f_{1}$ is an extension of $f$ to $A \cup \hat{C} \cup \hat{D}$ such that $f_{1}^{-1}(\{c\})=\hat{C}$ and $f_{1}^{-1}(\{d\})=\hat{D}$. The fact that $f_{1}$ is continuous follows from the "pasting lemma" ${ }^{1}$ of elementary topology applied to the functions $f: A \rightarrow[c, d], \mathbf{c}: \hat{C} \rightarrow\{c\}$, and $\mathbf{d}: \hat{D} \rightarrow\{d\}$. Since $A \cup \hat{C} \cup \hat{D}$ is closed, it follows from [TU] that there exists a continuous extension $\hat{f}_{1}: X \rightarrow[c, d]$ of $f_{1}$. Now if $\hat{f}_{1}^{-1}(\{c\})=\hat{C}$ and $\hat{f}_{1}^{-1}(\{d\})=\hat{D}$, we can set $\hat{f}=\hat{f}_{1}$ and be done. Therefore let us assume that the sets $C_{1}:=\hat{f}_{1}^{-1}(\{c\}) \backslash \hat{C}$ and $D_{1}:=\hat{f}_{1}^{-1}(\{d\}) \backslash \hat{D}$ are nonempty (later we will handle the case when exactly one of $C_{1}, D_{1}$ is empty).

Our strategy is to define a continuous function $\varphi$ on $X$ such that $\hat{f}=\hat{f}_{1}+\varphi$ satisfies the conclusion of the theorem. Clearly we must have $\varphi(A \cup \hat{C} \cup \hat{D})=\{0\},\left.\varphi\right|_{C_{1}}>0$, and $\left.\varphi\right|_{D_{1}}<0$ in order to make the proper adjustments to $\hat{f}_{1}$. Moreover, $\varphi$ should not be too large in magnitude, or else we will create unwanted points $x$ where $\hat{f}(x) \leq c$ or $\hat{f}(x) \geq d$.

To begin, observe that by hypothesis, $\hat{C}$ is closed $G_{\delta}$; hence we can write $\hat{C}=\bigcap_{n=1}^{\infty} G_{n}$ for a decreasing sequence $\left\{G_{n}\right\}$ of open sets. Moreover, by the normality of $X$, we may assume that $G_{n}^{c} \cap \overline{G_{n+1}}=$ $\emptyset$ for each $n$, where $G_{n}^{c}$ denotes the complement of $G_{n}$ and $\overline{G_{n+1}}$ denotes the closure of $G_{n+1}$. It follows from Urysohn's lemma that for each $n$, there exists a continuous function $\gamma_{n}: \hat{C} \cup C_{1} \rightarrow\left[0,2^{-n}\right\}$

${ }^{1}$ If $g_{1}$ and $g_{2}$ are continuous functions defined on closed sets $C_{1}$ and $C_{2}$, respectively, and if $g_{1}$ and $g_{2}$ agree on $C_{1} \cap C_{2}$, then they can be used to define a new function piecewise on $C_{1} \cup C_{2}$ (in the obvious way) which is continuous on $C_{1} \cup C_{2}$. 
such that

$$
\gamma_{n}\left(\left(\hat{C} \cup C_{1}\right) \cap G_{n}^{c}\right)=\left\{2^{-n}\right\} \quad \text { and } \quad \gamma_{n}\left(\left(\hat{C} \cup C_{1}\right) \cap \overline{G_{n+1}}\right)=\{0\}
$$

We now define the function $\gamma$ on $\hat{C} \cup C_{1}$ by

$$
\gamma(x):=\sum_{n=1}^{\infty} \gamma_{n}(x)
$$

Since for each $n, \gamma_{n}$ is continuous and $\left|\gamma_{n}\right| \leq 2^{-n}$, the above series converges uniformly and thus $\gamma: \hat{C} \cup C_{1} \rightarrow[0,1]$ is continuous. Clearly $\gamma$ is zero on $\hat{C}$ and strictly positive on $C_{1}$.

In a similar fashion, we can define a continuous function $\delta: \hat{D} \cup$ $D_{1} \rightarrow[-1,0]$ which is zero on $\hat{D}$ and strictly negative on $D_{1}$. It follows from the pasting lemma that the function $\alpha: A \cup \hat{C} \cup C_{1} \cup$ $\hat{D} \cup D_{1} \rightarrow[-1,1]$ given by

$$
\alpha(x):= \begin{cases}\gamma(x) & \text { if } x \in \hat{C} \cup C_{1} \\ \delta(x) & \text { if } x \in \hat{D} \cup D_{1} \\ 0 & \text { otherwise }\end{cases}
$$

is continuous. Our final step in the construction of $\varphi$ is to use [TU] to extend $\alpha$ to a continuous function $\hat{\alpha}: X \rightarrow[-1,1]$, and define

$$
\varphi:=\frac{\mathbf{c}-\hat{f}_{1}}{2} \vee \hat{\alpha} \wedge \frac{\mathbf{d}-\hat{f}_{1}}{2} .
$$

Observe that if $t_{1} \leq 0 \leq t_{2}$ for numbers $t_{1}$ and $t_{2}$, then $\left(t_{1} \vee t\right) \wedge t_{2}=$ $t_{1} \vee\left(t \wedge t_{2}\right)$ for any $t \in \mathbf{R}$, so no parentheses are needed in the above formula for $\varphi$. We claim that $\hat{f}:=\hat{f}_{1}+\varphi$ is the desired extension of $f$. It is clear that $\hat{f}$ is continuous and $\left.\hat{f}\right|_{A}=f$, because $\left.\varphi\right|_{A}=\mathbf{0}$. The following implications are also easily verified:

$$
x \in \hat{C} \Rightarrow \hat{\alpha}(x)=0 \Rightarrow \varphi(x)=0 \Rightarrow \hat{f}(x)=c
$$

$$
x \in \hat{D} \Rightarrow \hat{\alpha}(x)=0 \Rightarrow \varphi(x)=0 \Rightarrow \hat{f}(x)=d
$$


(3)

$$
\begin{aligned}
x \in C_{1} & \Rightarrow \hat{\alpha}(x)>0 \\
& \Rightarrow \hat{f}(x)=c+\left(\frac{c-c}{2} \vee \hat{\alpha}(x) \wedge \frac{d-c}{2}\right) \\
& =c+\left(\hat{\alpha}(x) \wedge \frac{d-c}{2}\right) \\
& \Rightarrow c<\hat{f}(x) \leq \frac{c+d}{2}<d \\
& \Rightarrow \hat{f}(x) \in(c, d)
\end{aligned}
$$

(4)

$$
\begin{aligned}
x \in D_{1} & \Rightarrow \hat{\alpha}(x)<0 \\
& \Rightarrow \hat{f}(x)=d+\left(\frac{c-d}{2} \vee \hat{\alpha}(x) \wedge \frac{d-d}{2}\right) \\
& =d+\left(\frac{c-d}{2} \vee \hat{\alpha}(x)\right) \\
& \Rightarrow c<\frac{c+d}{2} \leq \hat{f}(x)<d \\
& \Rightarrow \hat{f}(x) \in(c, d)
\end{aligned}
$$

(5) $x \in \hat{f}_{1}^{-1}((c, d)) \Rightarrow \hat{f}(x)=\hat{f}_{1}(x)$

$$
\begin{aligned}
& +\left(\frac{c-\hat{f}_{1}(x)}{2} \vee \hat{\alpha}(x) \wedge \frac{d-\hat{f}_{1}(x)}{2}\right) \\
\Rightarrow & c<\frac{c+\hat{f}_{1}(x)}{2} \leq \hat{f}(x) \leq \frac{d+\hat{f}_{1}(x)}{2}<d \\
\Rightarrow & \hat{f}(x) \in(c, d) .
\end{aligned}
$$

Thus $\hat{f}$ maps $X$ into $[c, d]$, with $\hat{f}^{-1}(\{c\})=\hat{C}$ and $\hat{f}_{1}(\{d\})=\hat{D}$, so $\hat{f}$ is the desired extension of $f$.

To treat the case when $C_{1}$ (respectively, $D_{1}$ ) is empty, we can construct $\alpha$ so that $\alpha$ is zero on $A \cup \hat{C} \cup \hat{D}$ and strictly positive on $C_{1}$ (respectively, zero on $A \cup \hat{C} \cup \hat{D}$ and strictly negative on $D_{1}$ ); the rest of the proof is essentially the same.

THEOREM 2. Let $X$ be a normal topological space, let $A$ be a closed subset of $X$, and let $f: A \rightarrow \mathbf{R}$ be continuous. Let 
$a_{1}, a_{2}, \ldots, a_{n}$ belong to $f(A)$ with $a_{1}<a_{2}<\cdots<a_{n}$, let $A_{i}:=$ $f^{-1}\left(\left\{a_{i}\right\}\right)$ for each $i$, and let

$$
\begin{aligned}
U_{0} & :=f^{-1}\left(\left(-\infty, \alpha_{1}\right)\right), U_{1}:=f^{-1}\left(\left(a_{1}, a_{2}\right)\right), \cdots U_{n-1} \\
& :=f^{-1}\left(\left(a_{n-1}, a_{n}\right)\right), U_{n}:=f^{-1}\left(\left(a_{n}, \infty\right)\right) .
\end{aligned}
$$

If $\hat{A}_{1}, \ldots, \hat{A}_{n}$ are disjoint closed $G_{\delta}$-sets in $X$ such that $\hat{A}_{i} \cap A=$ $A_{i}$ for each $i$ and $X \backslash\left(\bigcup_{i=1}^{n} \hat{A}_{i}\right)=\bigcup_{i=0}^{n} \hat{U}_{i}$, where $\hat{U}_{0}, \ldots, \hat{U}_{n}$ are disjoint open sets satisfying $U_{i} \cap A=U_{i}$ for each $i$, then there exists a continuous extension $\hat{f}$ of $f$ to all of $X$ such that $\hat{f}^{-1}\left(\left\{a_{i}\right\}\right)=\hat{A}_{i}$ for each $i$.

Proof. For $i=1, \ldots, n-1$, let $f_{i}:=\left.f\right|_{A_{i} \cup U_{i} \cup A_{i+1}}$. By Theorem 1 , there exist continuous extensions $\hat{f}_{i}: \hat{A}_{i} \cup \hat{U}_{i} \cup \hat{A}_{i+1} \rightarrow\left[a_{i}, a_{i+1}\right]$ of each function $f_{i}$, such that $\hat{f}_{i}^{-1}\left(\left\{a_{i}\right\}\right)=\hat{A}_{i}$ and $\hat{f}_{i}^{-1}\left(\left\{a_{i+1}\right\}\right)=$ $\hat{A}_{i+1}$. Moreover, $f_{0}:=\left.f\right|_{A_{1} \cup U_{0}}$ and $f_{n}:=\left.f\right|_{A_{n} \cup U_{n}}$ can similarly be extended to continuous functions $\hat{f}_{0}: \hat{A}_{1} \cup \hat{U}_{0} \rightarrow\left(-\infty, a_{1}\right]$ and $\hat{f}_{n}$ : $\hat{A}_{n} \cup \hat{U}_{n} \rightarrow\left[a_{n}, \infty\right)$ such that $\hat{f}_{0}^{-1}\left(\left\{a_{1}\right\}\right)=\hat{A}_{1}$ and $\hat{f}_{n}^{-1}\left(\left\{a_{n}\right\}\right)=\hat{A}_{n}$. To see that this is true, consider (say) $f_{n}$. As in the proof of the Theorem 1, we can extend $f_{n}$ to a continuous function $\left(\hat{f}_{n}\right)_{1}: \hat{A}_{n} \cup$ $\hat{U}_{n} \rightarrow\left[a_{n}, \infty\right)$ such that $\hat{A}_{n} \subseteq\left(\hat{f}_{n}\right)_{1}^{-1}\left(\left\{a_{n}\right\}\right) ;$ if $\left(\hat{f}_{n}\right)_{1}^{-1}\left(\left\{a_{n}\right\}\right) \neq \hat{A}_{n}$, then we can remedy this by adding to $\left(\hat{f}_{n}\right)_{1}$ a continuous function $\gamma: \hat{A}_{n} \cup \hat{U}_{n}: \rightarrow\left[a_{n}, \infty\right)$ similar to that constructed in the proof of Theorem 1 , which is zero on $\hat{A}_{n}$ and strictly positive on $\hat{U}_{n}$, so that $\hat{f}_{n}:=\left(\hat{f}_{n}\right)_{1}+\gamma$ is the desired extension of $f_{n}$. The extension of $f_{0}$ is handled similarly, using a negative analog of $\gamma$.

To complete the proof, we can simply define $f$ piecewise on $X$ in the obvious way (for example, let $\left.\hat{f}\right|_{\hat{A}_{0} \cap \hat{U}_{0}}=\hat{f}_{0}$ ), and note that $\hat{f}$ is continuous on $X$ by the pasting lemma.

REMARK. It is easy to see that continuity in the above theorems cannot be replaced by uniform continuity or bounded uniform continuity. For example, let $X:=\mathbf{R}^{2}, A:=\mathbf{R} \times\{0\}, f(x, y):=$ $|x| \wedge 1, A_{1}:=f^{-1}(\{0\}):=\{(0,0)\}, \hat{A}_{1}:=A_{1} \cup\{(x, y) \mid y=1 / x\}$. Then $f$ is bounded and uniformly continuous on $A$, but $f$ cannot be extended to a uniformly continuous function $\hat{f}$ on $X$ such that $\hat{f}^{-1}(\{0\})=A_{1}$. 
2. Extending products. Let $X$ be a normal topological space, let $A$ be a closed subset of $X$, and let $f, g: A \rightarrow \mathbf{R}$ be continuous. By [TU], there exist continuous extensions $\hat{f}$ and $\hat{g}$ of $f$ and $g$ to all of $X$. Obviously, in extending $f$ and $g$, we also create a continuous extension $\hat{f} \hat{g}$ of the product $f g$. It is therefore natural to ask whether, given a continuous function $h: X \rightarrow \mathbf{R}$ such that $f g=\left.h\right|_{A}$, we can find continuous extensions $\hat{f}$ and $\hat{g}$ to all of $X$ such that $\hat{f} \hat{g}=h$.

We begin with two examples which show that, in general, such extensions may fail to exist. These examples are illustrated in Figures 1 and 2 below. In each case, we take $X$ to be a square in $\mathbf{R}^{2}$, with $A$ being the closed left half of $X$, and it is easy to see that we can find continuous functions $f, g$, and $h$ with the values indicated in the figures.

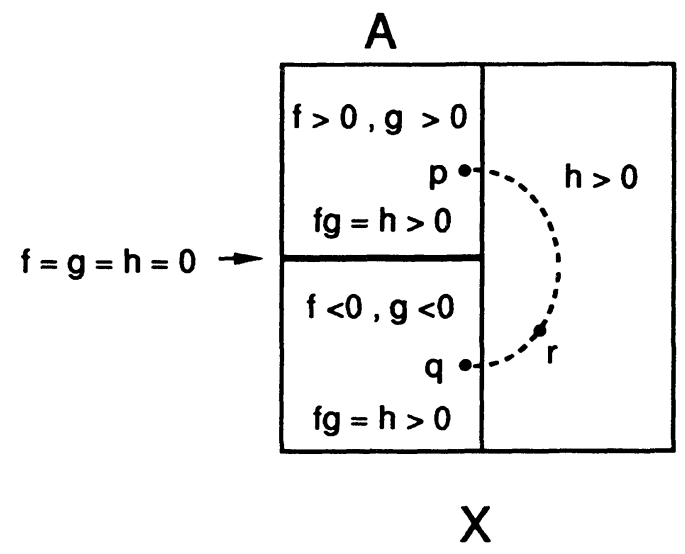

FiguRE 1.

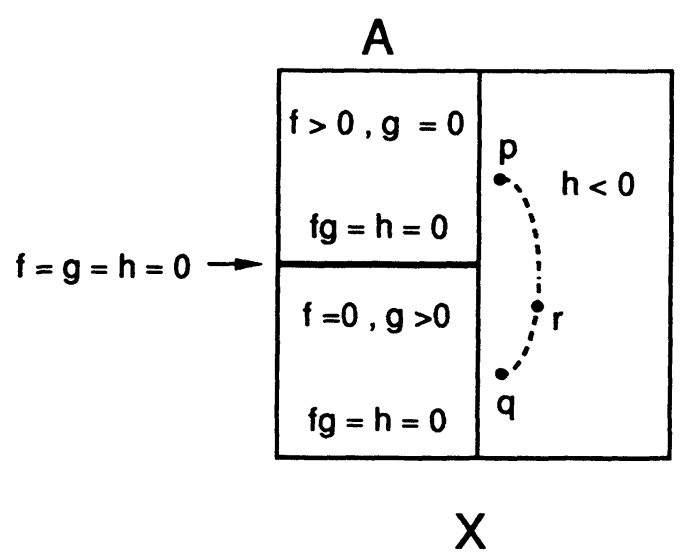

FiguRE 2. 
Suppose that continuous extensions $\hat{f}$ and $\hat{g}$ of $f$ and $g$ existed in Figure 1. Since $\hat{f}(p)>0$ and $\hat{f}(q)<0$ (with $p$ and $q$ as indicated), there must be a point $r$ outside $A$ on the dotted curve at which $\hat{f}(r)=0$. But then we cannot have $\hat{f}(r) \hat{g}(r)=h(r)>0$.

For the second case, suppose that continuous extensions $\hat{f}$ and $\hat{g}$ of $f$ and $g$ existed in Figure 2, such that $\hat{f} \hat{g}=h$. We could then find points $p$ and $q$ as indicated, with $\hat{f}(p)>0$ and $\hat{g}(q)>0 \Rightarrow \hat{f}(q)<0$ since $h(q)<0$. Again there must be a point $r$ outside $A$ on the dotted curve such that $\hat{f}(r)=0$, which contradicts $\hat{f}(r) \hat{g}(r)=$ $h(r)<0$.

In the first case, we had the simple relationship $g^{-1}(\{0\})=$ $f^{-1}(\{0\})$ between zero sets, but the mere fact that both $f$ and $g$ were allowed to take on both positive and negative values allowed us to preclude the possibility of suitable extensions. In the second case, both $f$ and $g$ were nonnegative, but by choosing their zero sets carefully, we were able to preclude the existence of the extensions again. Thus even when $X$ is a compact metric space, the desired extensions are not guaranteed. However, our next theorem shows that the above cases are, roughly speaking, the worst possible; that is, by requiring that at least one of $f, g$ be nonnegative (or nonpositive), and by demanding an inclusion relation on their zero sets, we obtain a sufficient condition for the existence of the desired extensions.

ThEOREM 3. Let $(X, d)$ be a compact metric space, and let $A$ be a closed subset of $X$. Suppose that $f, g: A \rightarrow \mathbf{R}$ and $h: X \rightarrow \mathbf{R}$ are continuous, with $g \geq 0$ (or $g \leq 0$ ), such that $f g=\left.h\right|_{A}$. If $g^{-1}(\{0\}) \subseteq f^{-1}(\{0\})$, then there exist continuous extensions $\hat{f}$ of $f$ and $\hat{g}$ of $g$ to all of $X$ such that $\hat{f} \hat{g}=h$.

Proof. For definiteness we will consider the case $g \geq 0$. First note that if $g>0$ (i.e. if $g$ is strictly positive on $A$ ), then we can apply [TU] to extend $g$ to a continuous function $\hat{g}>0$ on all of $x$ (by using $\hat{g}+d(x, A)$ if necessary). In the case, defining $\hat{f}:=h / \hat{g}$ completes the proof.

Therefore let us assume that $g^{-1}(\{0\}) \neq \emptyset$. Defining $Z:=$ $h^{-1}(\{0\})$, we have by hypothesis that

$$
Z \cap A=f^{-1}(\{0\}) \supseteq g^{-1}(\{0\}) \neq \emptyset .
$$

Using [TU], let $\hat{g}_{1} \geq 0$ be a continuous extension of $g$ to all of $X$. Again, by adding the function $d(x, A)$ to $\hat{g}_{1}$ if necessary, we 
may assume that $\hat{g}_{1}>0$ on $X \backslash A$. The function $\hat{g}_{1}$ is our first approximation to a suitable extension of $g$; our first approximation to a suitable extension of $f$ is the function $\hat{f}_{1}$ defined by

$$
\hat{f}_{1}(x):= \begin{cases}f(x) & \text { if } x \in A \\ h(x) / \hat{g}_{1}(x) & \text { if } x \in X \backslash A .\end{cases}
$$

Since $\left.\hat{g}_{1}\right|_{A}=g$, we have $\hat{f}_{1}(x)=h(x) / \hat{g}_{1}(x)$ whenever $g(x) \neq 0$, so $\hat{f}_{1}$ is continuous at all points of $X \backslash g^{-1}(\{0\})$. Moreover, if a point $x_{0} \in g^{-1}(\{0\})$ belongs to the interior of $A \cup Z$, then $\hat{f}_{1}$ is continuous at $x_{0}$, because $\left.\hat{f}_{1}\right|_{A \cup Z}$ is continuous on $A \cup Z$ (this follows from the pasting lemma applied to the functions $f: A \rightarrow \mathbf{R}$ and $\mathbf{0}: A \rightarrow 0$ ). Hence the only type of point at which $\hat{f}_{1}$ might not be continuous is a point $x_{0} \in g^{-1}(\{0\}) \cap \partial(A \cup Z)$, where $\partial(A \cup Z)$ denotes the boundary of $A \cup Z$.

From now on, we consider only those $n$ large enough so that the sets

$$
H_{n}:=\left\{x \in X|| h(x) \mid \geq 4^{-(n+1)}\right\}
$$

are nonempty. Since $X$ is compact, $h$ and $\hat{g}_{1}$ are uniformly continuous, and since each $H_{n}$ is compact, $\hat{g}_{1}$ is bounded away from zero on $H_{n}$. It follows that $h / \hat{g}_{1}$ is uniformly continuous on $H_{n}$. Moreover, we can find a decreasing sequence of positive real numbers $\delta_{n}$ which satisfy the following rules:

$$
\begin{gathered}
\delta_{n}<\inf \left\{d(x, y) \mid x \in H_{n}, y \in\left(H_{n+1}\right)^{c}\right\} \\
\delta_{n}<\inf \left\{d(x, y) \mid x \in H_{n}, y \in Z\right\}
\end{gathered}
$$

(3) $\left(x, y \in H_{n+1}\right.$ and $\left.d(x, y)<\delta_{n}\right)$

$$
\Rightarrow\left|h(x) / \hat{g}_{1}(x)-h(y) / \hat{g}_{1}(y)\right|<2^{-(n+1)} .
$$

Next, for each $n$, we define the set $A_{n}$ by

$$
A_{n}:=\left\{x \in X \mid d(x, A) \geq \delta_{n} / 2\right\} .
$$

Now $A \cup Z$ and $A_{n} \cap H_{n}$ are disjoint closed sets for each $n$, so we can find a function $\gamma_{n}: X \rightarrow\left[0,2^{-n}\right]$ for each $n$ which is zero on 
$A \cup Z$ and equal to $2^{-n}$ on $A_{n} \cap H_{n}$. As in the proof of Theorem 1 , the function $\gamma: X \rightarrow[0,1]$ given by

$$
\gamma(x):=\sum_{n=1}^{\infty} \gamma_{n}(x)
$$

is continuous, and $\gamma(A \cup Z)=\{0\}$. We claim that the function $\hat{f}$ and $\hat{g}$ given by

$$
\hat{g}:=\hat{g}_{1}+\gamma \text { and } \hat{f}(x):= \begin{cases}f(x) & \text { if } x \in A \\ h(x) / \hat{g}(x) & \text { if } x \in X \backslash A\end{cases}
$$

are the desired extensions of $f$ and $g$.

It is easy to check that: $\hat{g}$ is continuous on $X,\left.\hat{g}\right|_{A}=g,\left.\hat{f}\right|_{A}=f$ and $\hat{f} \hat{g}=h$. As in our previous remarks about $\hat{f}_{1}, \hat{f}$ is continuous everywhere, except possibly at points of $g^{-1}(\{0\}) \cap \partial(A \cup Z)$. Therefore let $x_{0} \in g^{-1}(\{0\}) \cap \partial(A \cup Z)$, let $\epsilon>0$, and choose $n \in \mathbf{N}$ such that $2^{-n}<\epsilon$. Since $\left.\hat{f}_{1}\right|_{A \cup Z}$ is continuous, we may choose $n$ large enough $\left(\delta_{n}\right.$ small enough) so that

$$
d\left(x, x_{0}\right)<\delta_{n} \Rightarrow\left|\hat{f}_{1}(x)\right|=\left|\hat{f}_{1}(x)-\hat{f}_{1}\left(x_{0}\right)\right|<\epsilon / 2
$$

for all $x \in A \cup Z$. Let $B_{0}$ be a ball centered at $x_{0}$ with radius $<\delta_{n} / 2$. Since $\gamma(A \cup Z)=\{0\}$, we have $\left.\hat{f}\right|_{A \cup Z}=\left.\hat{f}_{1}\right|_{A \cup Z}$, so by (4),

$$
x \in B_{0} \cap(A \cup Z) \Rightarrow\left|\hat{f}(x)-\hat{f}\left(x_{0}\right)\right|=\left|\hat{f}_{1}(x)-\hat{f}_{1}\left(x_{0}\right)\right|<\epsilon / 2<\epsilon .
$$

Suppose now that $x \in B_{0} \backslash(A \cup Z)$; then since $H_{m} \uparrow X \backslash Z$, there is a smallest $m$ such that $x \in H_{m}$, and $m>n$ by (2), because $d(x, Z)<\delta_{n}$. We have $4^{-(m+1)} \leq|h(x)|<4^{-m}$. Suppose that $x$ also belongs to $A_{m}$, and hence to $A_{m} \cap H_{m}$. Then since $\hat{f}\left(x_{0}\right)=0$,

$$
\begin{aligned}
\left|\hat{f}(x)-\hat{f}\left(x_{0}\right)\right| & =|\hat{f}(x)|=\frac{|h(x)|}{\hat{g}(x)}<\frac{|h(x)|}{\gamma_{m}(x)}<\frac{4^{-m}}{2^{-m}} \\
& =2^{-m}<2^{-n}<\epsilon .
\end{aligned}
$$

On the other hand, if $x \notin A_{m}$, then there exists $y \in A$ such that $d(x, y)<\delta_{m} / 2$. Moreover, $\delta_{m} / 2<\inf \left\{d(w, z) \mid w \in H_{m}, z \in\right.$ 
$\left.\left(H_{m+1}\right)^{c}\right\}$ by (1), so $y$ must belong to $H_{m+1}$. Since also $x \in H_{m} \subset$ $H_{m+1}$, we have from (3) that

$$
\begin{aligned}
\left|\hat{f}(x)-\hat{f}\left(x_{0}\right)\right| & =|\hat{f}(x)|=|h(x) / \hat{g}(x)| \leq\left|h(x) / \hat{g}_{1}(x)\right| \\
& \leq\left|h(x) / \hat{g}_{1}(x)-h(y) / \hat{g}_{1}(y)\right|+\left|h(y) / \hat{g}_{1}(y)\right| \\
& <2^{-(m+1)}+\left|\hat{f}_{1}(y)\right|<\epsilon / 2+\left|\hat{f}_{1}(y)\right| .
\end{aligned}
$$

To estimate $\left|\hat{f}_{1}(y)\right|$, note that

$$
d\left(y, x_{0}\right) \leq d(y, x)+d\left(x, x_{0}\right)<\delta_{m} / 2+\delta_{n} / 2<\delta_{n},
$$

so (4) implies that $\left|\hat{f}_{1}(y)\right|<\epsilon / 2$, and thus from (5),

$$
\left|\hat{f}(x)-\hat{f}\left(x_{0}\right)\right|<\epsilon / 2+\epsilon / 2=\epsilon .
$$

Hence we have shown that $x \in B_{0} \Rightarrow\left|\hat{f}(x)-\hat{f}\left(x_{0}\right)\right|<\epsilon$, so $\hat{f}$ is continuous at $x_{0}$. It follows that $\hat{f}$ is continuous on $X$ and that $\hat{f}$ and $\hat{g}$ are the desired extensions of $f$ and $g$.

Corollary 4. Let $(X, d), A$ and $h$ be as in Theorem 3. Let $f, g_{1}, \ldots, g_{n}: A \rightarrow \mathbf{R}$ be continuous functions with $g_{i} \geq 0$ or $g_{i} \leq 0$ and

$$
f^{-1}(\{0\}) \supseteq g_{n}^{-1}(\{0\}) \supseteq \cdots \supseteq g_{1}^{-1}(\{0\}),
$$

such that $f g_{1} \cdots g_{n}=\left.h\right|_{A}$. Then there exists a continuous extension $\hat{f}$ of $f$ and a continuous extension $\hat{g}_{i}$ of each $g_{i}$ to all of $X$ such that $\hat{f} \hat{g}_{1} \cdots \hat{g}_{n}=h$.

Proof. The proof goes by induction on $n$. Suppose the theorem is true for any $n-1$ functions $g_{1}, \ldots, g_{n-1}$ as described above (we know it is true for a single $g$ by Theorem 3). Since each of the $g_{i}$ is continuous and nonnegative (or nonpositive), their product is also. Hence by Theorem 3 , there exists a continuous extension $\hat{f}$ of $f$ and a continuous extension $\hat{G}$ of the product $g_{1} \cdots g_{n}$ to all of $X$ such that $\hat{f} \hat{G}=h$. Then $\left(g_{1} \cdots g_{n-1}\right) g_{n}=\left.\hat{G}\right|_{A}$, and by hypothesis there exist continuous extensions $\hat{g}_{i}$ of each $g_{i}$ to all of $X$ such that $\hat{g}_{1} \cdots \hat{g}_{n}=\hat{G}$. Hence $\hat{f} \hat{g}_{1} \cdots \hat{g}_{n}=h$.

REMARKS. The problems considered in this section are special cases of a much more general problem, which we will now formulate. 
Let $A$ be a closed subset of a normal topological space $X$, and let $P\left(u_{1}, \ldots, u_{n}\right)$ be a real-valued function of $n$ real variables. Suppose we have $n$ continuous functions $f_{1}, \ldots, f_{n}: A \rightarrow \mathbf{R}$ and a continuous function $h: X \rightarrow \mathbf{R}$ such that

$$
h(x)=P\left(f_{1}(x), \ldots, f_{n}(x)\right) \quad \text { for each } x \in A .
$$

The question we wish to pose is: Do there exist continuous extensions $\hat{f}_{1}, \ldots, \hat{f}_{n}: X \rightarrow \mathbf{R}$ of the $f_{i}$ such that $(*)$ holds for all $x \in X$ ? As we shall see, certain special cases of this problem have interesting interpretations. Let us note first that we have just explored the cases when $P\left(u_{1}, u_{2}\right)=u_{1} u_{2}$ and $P\left(u_{1}, \ldots, u_{n}\right)=u_{1} u_{2} \cdots u_{n}$. Also, the problem is an easy one if $P\left(u_{1}, \ldots, u_{n}\right)=u_{1}+\cdots+u_{n}$. We merely use [TU] to extend $f_{2}, \ldots, f_{n}$ to $\hat{f}_{2}, \ldots, \hat{f}_{n}$ and define $\hat{f}_{1}(x):=h(x)-\left(\hat{f}_{2}(x)+\cdots+\hat{f}_{n}(x)\right)$. The case when $P\left(u_{1}, u_{2}\right)=$ $u_{1} / u_{2}$ is also easy if $f_{2}$ is of constant sign (say $f_{2}>0$ ) and $A$ is a $G_{\delta}$-set; we extend $f_{2}$ to $\hat{f}_{2}>0$ and define $\hat{f}_{1}(x):=h(x) \hat{f}_{2}(x)$. There are many other cases, however, for which the answers are not clear.

Some important special cases occur if we put restrictions on $h$ and perhaps on the functions $f_{1}, \ldots, f_{n}$ and their extensions. For example, suppose we have $P\left(u_{1}, \ldots, u_{n}\right)=u_{1}+\cdots+u_{n}$ and $h=\mathbf{1}$, and we demand that the functions $f_{1}, \ldots, f_{n}$ and their extensions be nonnegative. Then the above question is equivalent to asking: If a finite partition of unity is defined on $A$, can it be extended to a partition of unity on all of $X$ ? Of course there is usually a topological restriction on a partition of unity, namely that it be subordinate to some predetermined open covering, and thus we might want the extended collection $\left\{\hat{f}_{1}, \ldots, \hat{f}_{n}\right\}$ to be subordinate to some predetermined open covering of $X$. This problem is formulated more precisely and solved in Section 4.

Another special case of interest is the case when $P\left(u_{1}, \ldots, u_{n}\right)=$ $\sum_{i \neq j}\left|u_{i} u_{j}\right|$. If we take $h=\mathbf{0}$, then the condition is the same as requiring that $\left|f_{i}\right| \wedge\left|f_{j}\right|=0$ whenever $i \neq j$, which is equivalent to saying that the collection $\left\{f_{1}, \ldots, f_{n}\right\}$ of functions is "pairwise disjoint", a property which is often useful in analysis. Our goal is then to find a pairwise disjoint set of extensions $\left\{\hat{f}_{1}, \ldots, \hat{f}_{n}\right\}$. This problem is solved in the next section. 
3. Extending pairwise disjoint collections. Recall that two continuous functions $f_{1}$ and $f_{2}$ are disjoint if $\left|f_{1}\right| \wedge\left|f_{2}\right|=\mathbf{0}$. An equivalent statement in terms of their supports $F_{1}$ and $F_{2}$ is that $\operatorname{int}\left(F_{1} \cap F_{2}\right)=\emptyset$. We have the following proposition for extending finite collections of pairwise disjoint continuous functions:

Proposition 5. Let $A$ be a closed subset of a normal space $X$, and let the functions $f_{1}, \ldots, f_{n}: A \rightarrow \mathbf{R}$ be continuous and pairwise disjoint. Then there exist pairwise disjoint continuous extensions $\hat{f}_{1}, \ldots, \hat{f}_{n}$ of the respective $f_{i}$ to all of $X$.

Proof. It will suffice to consider the case when $f_{1}, \ldots, f_{n} \geq 0$. For each $1 \leq k \leq n$ we can find a continuous extension $g_{k}: X \rightarrow[0, \infty)$ of $f_{k}$, by [T्U]. For each such $k$, define $\hat{f}_{k}$ by

$$
\hat{f}_{k}(x):=\left(g_{k}(x)-\bigvee_{i \neq k} g_{i}(x)\right) \vee 0 .
$$

Clearly each $\hat{f}_{k}$ is continuous. To show that $\left.\hat{f}_{k}\right|_{A}=f_{k}$ for each $k$, let $a \in A$. From the above definition, we see that

$$
f_{k}(a)=g_{k}(a)=0 \Rightarrow \hat{f}_{k}(a)=0 .
$$

On the other hand, if $f_{k}(a)>0$, then by disjointness condition, $g_{i}(a)=f_{i}(a)=0$ for all $i \neq j$, so $\hat{f}_{k}(a)=g_{k}(a)=f_{k}(a)$.

Finally, to show the pairwise disjointness of the $\hat{f}_{i}$, fix $1 \leq j, k \leq$ $n$ with $j \neq k$. Then

$$
\hat{f}_{k}(x)>0 \Rightarrow g_{j}(x)<g_{k}(x) \Rightarrow \hat{f}_{j}(x)=0 .
$$

An obvious question at this point would be whether we can extend an infinite collection of pairwise disjoint continuous functions. The answer is affirmative in the case of a countable collection, but the proof is rather technical and will be included in a later work. For the case of an arbitrary infinite collection we do not know the answer, but we do know that arbitrary collections can be extended in the case when $X$ is a metric space. This is proved in the following proposition, which employs a construction used by Mandelkern in 
[M], and which is similar to ones used by F. Riesz in [Ke], Tietze in $[\mathbf{T}]$, and Hausdorff in $[\mathbf{H}]$.

Proposition 6. Let $A$ be a closed subset of a metric space $(X, d)$, and let $\left\{f_{\gamma}\right\}_{\gamma \in \Gamma}$ be a set of pairwise disjoint continuous functions from $A$ to $\mathbf{R}$. Then there exists a set $\left\{\hat{f}_{\gamma}\right\}_{\gamma \in \Gamma}$ of pairwise disjoint continuous functions from $X$ to $\mathbf{R}$, such that $\left.\hat{f}_{\gamma}\right|_{A}=f_{\gamma}$ for each $\gamma \in \Gamma$.

Proof. Again it will suffice to consider the case when $f_{\gamma} \geq 0$ for each $\gamma \in \Gamma$. For each $\gamma$, let $\varphi_{\gamma}: A \rightarrow[1,2)$ be the function $\varphi_{\gamma}(x):=\frac{2}{\pi} \arctan \left(f_{\gamma}(x)\right)+1$, so that $\varphi_{\gamma}(x)=1 \Leftrightarrow f_{\gamma}(x)=0$. We are done if we can find a continuous extension $\hat{\varphi}_{\gamma}: X \rightarrow[1,2)$ of each $\varphi_{\gamma}$ such that $\hat{\varphi}_{\alpha} \wedge \hat{\varphi}_{\beta}=1$ whenever $\alpha \neq \beta$; for then we can define $\hat{f}_{\gamma}$ by $\hat{f}_{\gamma}(x):=\tan \left(\frac{\pi}{2}\left(\hat{\varphi}_{\gamma}(x)-1\right)\right)$, and have $\left|\hat{f}_{\alpha}\right| \wedge\left|\hat{f}_{\beta}\right|=\mathbf{0}$ whenever $\alpha \neq \beta$.

Folowing the construction used in $[\mathbf{M}]$, we define $\hat{\varphi}_{\gamma}$ for each $\gamma$ by $\hat{\varphi}_{\gamma}(x):=\varphi_{\gamma}(x)$ if $x \in A$, and

$$
\hat{\varphi}_{\gamma}(x):=\inf _{a \in A} \varphi_{\gamma}(a) \frac{d(x, a)}{d(x, A)} \quad \text { if } x \notin A .
$$

It is clear that $\hat{\varphi}_{\gamma}: X \rightarrow[1,2]$. If $\hat{\varphi}_{\gamma}^{-1}(\{2\}) \neq \emptyset$, then we can remedy this by taking the infimum of $\hat{\varphi}_{\gamma}$ with a continuous function which is equal to 1 and 2 , respectively, on the disjoint closed sets $\hat{\varphi}_{\gamma}^{-1}(\{2\})$ and $A$. Thus we may assume that $\hat{\varphi}_{\gamma}: X \rightarrow[1,2)$.

Now in $[\mathbf{M}]$ it. is shown that if $\varphi_{\gamma}$ is uniformly continuous, then so is $\hat{\varphi}_{\gamma}$. With only slight modifications, the same proof applies if we replace uniform continuity with continuity. Hence we only need show, as mentioned above, that $\hat{\varphi}_{\alpha} \wedge \hat{\varphi}_{\beta}=1$ whenever $\alpha \neq \beta$.

Clearly this true at all points of $A$, so fix $x \in X \backslash A$, and choose two distinct $\alpha, \beta \in \Gamma$. For each $\gamma$ let $G_{\gamma}$ be the set $G_{\gamma}:=\{a \in$ $\left.A \mid \varphi_{\gamma}(a)>1\right\}$. Let us fix $x$ and $\alpha$. Then $d\left(x, G_{\alpha}\right) \geq d(x, A)$. If $d\left(x, G_{\alpha}\right)>d(x, A)$, then $d\left(x, A \backslash G_{\alpha}\right)=d(x, A)$, and since $\varphi_{\alpha}(a)=1$ for each $a \in A \backslash G_{\alpha}$, we have

$$
\hat{\varphi}_{\alpha}(x) \leq \inf _{a \in A \backslash G_{\alpha}} \varphi_{\alpha}(a) \frac{d(x, a)}{d(x, A)}=\inf _{a \in A \backslash G_{\alpha}} \frac{d(x, a)}{d(x, A)}=1,
$$

so $\hat{\varphi}_{\alpha}(x)=1$. On the other hand, if $d\left(x, G_{\alpha}\right)=d(x, A)$, then using the fact that $\varphi_{\beta}(a)=1$ for each $a \in G_{\alpha}$ (by the pairwise disjointness 
of $f_{\alpha}$ and $f_{\beta}$ ), we have

$$
\hat{\varphi}_{\beta}(x) \leq \inf _{a \in G_{\alpha}} \varphi_{\beta}(a) \frac{d(x, a)}{d(x, A)}=\inf _{a \in G_{\alpha}} \frac{d(x, a)}{d(x, A)}=1,
$$

so $\hat{\varphi}_{\beta}(x)=1$. Thus $\hat{\varphi}_{\alpha} \wedge \hat{\varphi}_{\beta}=1$, and the proposition is proved.

4. Extending partitions of unity. The following theorem affirmatively answers a natural question on extending partitions of unity.

THEOREM 7. Let $A$ be a closed subset of a normal topological space $X$, and let $f_{1}, \ldots, f_{n}: A \rightarrow[0,1]$ form a partition of unity subordinate to an open covering $\left\{U_{i}\right\}_{i=1}^{n}$ of $A$. If $\left\{\hat{U}_{i}\right\}_{i=1}^{n}$ is an open covering of $X$ such that $\hat{U}_{i} \cap A=U_{i}$ for each $i$, then there exists a partition of unity $\left\{\hat{f}_{i}\right\}_{i=1}^{n}$ on $X$ subordinate to $\left\{\hat{U}_{i}\right\}_{i=1}^{n}$ such that $\left.\hat{f}_{i}\right|_{A}=f_{i}$ for each $i$.

Proof. Let us put $F_{i}:=\operatorname{supp}\left(f_{i}\right)$ for each $i$, and define $A_{1}:=$ $F_{1} \cup\left(\hat{U}_{2} \cup \cdots \cup \hat{U}_{n}\right)^{c}$. Then $A_{1} \subset \hat{U}_{1}$ and we can find an open set $V_{1}$ with $A_{1} \subset V_{1} \subset \overline{V_{1}} \subset \hat{U}_{1}$. Moreover, $\left\{V_{1}, \hat{U}_{2}, \ldots, \hat{U}_{n}\right\}$ covers $X$. Inductively, given an open covering $\left\{V_{1}, \ldots, V_{k-1}, \hat{U}_{k}, \ldots, \hat{U}_{n}\right\}$ of $X$, let $A_{k}:=F_{k} \cup\left(V_{1} \cup \cdots \cup V_{k-1} \cup \hat{U}_{k+1} \cup \cdots \cup \hat{U}_{n}\right)^{c}$, and choose an open set $V_{k}$ with $A_{k} \subset V_{k} \subset \overline{V_{k}} \subset \hat{U}_{k}$. At the $n$th step of the process, we obtain an open covering $\left\{V_{i}\right\}_{i=1}^{n}$ of $X$ with $F_{i} \subset V_{i} \subset \overline{V_{i}} \subset \hat{U}_{i}$ for each $i$. Similarly, starting with $\left\{V_{i}\right\}_{i=1}^{n}$, we can find an open covering $\left\{W_{i}\right\}_{i=1}^{n}$ of $X$ such that $F_{i} \subset W_{i} \subset \overline{W_{i}} \subset V_{i}$ for each $i$.

Next we extend each $f_{i}$ to a continuous function $g_{i}: X \rightarrow[0,1]$, and we choose a continuous function $\gamma_{i}: X \rightarrow[0,1]$ for each $i$ such that $\gamma_{i}\left(\overline{W_{i}}\right)=\{1\}$ ad $\gamma_{i}\left(V_{i}^{c}\right)=\{0\}$. Then it is easy to check that $F_{i} \subset \operatorname{supp}\left(g_{i} \gamma_{i}\right) \subset \hat{U}_{i}$ and $\left.g_{i} \gamma_{i}\right|_{A}=f_{i}$. If we had $\sum g_{i} \gamma_{i}>0$, we could define $\hat{f}_{k}:=g_{k} \gamma_{k} /\left(\sum g_{i} \gamma_{i}\right)$ and be done. However, since this may not be the case, we make the following adjustment.

Let $B:=\left(\sum g_{i} \gamma_{i}\right)^{-1}(\{1\})$. Then $B$ is a closed $G_{\delta}$-set containing $A$ and we can find, as in previous proofs, a nonnegative continuous function $\beta$ on $X$ which is zero on $B$ and strictly positive on $B^{c}$. For each $i$, we now define

$$
\hat{g}_{i}:=g_{i} \gamma_{i}+\beta \gamma_{i}
$$


Since $\hat{g}_{i}$ is a multiple of $\gamma_{i}$, we have supp $\hat{g}_{i} \subset \overline{V_{i}} \subset U_{i}$ for each $i$. To show that $\sum \hat{g}_{i}>0$, observe that if $x \in B$ then $\beta(x)=0$, so $\sum \hat{g}_{i}(x)=\sum g_{i}(x) \gamma_{i}(x)=1$. On the other hand, if $x \notin B, x$ still belongs to $W_{k}$ for some $k$, and thus $\sum \hat{g}_{i}(x) \geq \hat{g}_{k}(x)>0$, because $\beta(x) \gamma_{k}(x)=\beta(x)>0$.

Finally, we can define, for $k=1, \ldots, n$,

$$
\hat{f}_{k}:=\frac{\hat{g}_{k}}{\sum \hat{g}_{i}}
$$

Clearly $\hat{f}_{k}$ is continuous and $\sum \hat{f}_{i}=\mathbf{1}$. Also, $\operatorname{supp}\left(\hat{f}_{k}\right)=\operatorname{supp}\left(\hat{g}_{k}\right) \subset$ $\hat{U}_{k}$, and $\hat{f}_{k}$ is an extension of $f_{k}$, because $A \subset B$ implies

$$
\left.\hat{f}_{k}\right|_{A}=\frac{\left.\hat{g}_{k}\right|_{A}}{\left.\sum \hat{g}_{i}\right|_{A}}=\frac{\left.g_{k} \gamma_{k}\right|_{A}}{\left.\sum g_{i} \gamma_{i}\right|_{A}}=\frac{f_{k}}{\sum f_{i}}=f_{k}
$$

REMARK. Again the obvious question: Does Theorem 7 hold for an infinite partition of unity?

5. Extending equicontinuous sets of functions. In this section we will generalize the range space to be a locally convex metric linear space $(Y, \varrho)$ over the real or complex field. Recall that for a metrizable $Y$, we can always make the additional assumption that the metric $\varrho$ is translation-invariant, i.e., $\varrho(\vec{y}+\vec{w}, \vec{z}+\vec{w})=\varrho(\vec{y}, \vec{z})$ for all $\vec{y}, \vec{w}, \vec{z} \in Y$.

Let us recall that a collection $\left\{f_{\gamma}\right\}_{\gamma \in \Gamma}$ of functions from a metric space $(X, d)$ to a metric space $(Y, \varrho)$ is called equicontinuous at a point $x \in X$ if, for any $\epsilon>0$ there exists $\delta>0$ such that $\varrho\left(f_{\gamma}\left(x^{\prime}\right), f_{\gamma}(x)\right)<\epsilon$ for all $\gamma \in \Gamma$ whenever $d\left(x^{\prime}, x\right)<\delta$. The collection is equicontinuous if it is equicontinuous at every point of $X$. It is in keeping with the spirit of preceding theorems to ask: If $A$ is a closed subset of a metric space $(X, d)$, and if $\mathcal{F}:=\left\{f_{\gamma}\right\}_{\gamma \in \Gamma}$ is an equicontinuous subset of $C(A, Y)$, does there exist an equicontinuous subset $\hat{\mathcal{F}}:=\left\{\hat{f}_{\gamma}\right\}_{\gamma \in \Gamma}$ of $C(X, Y)$ such that $\left.\hat{f}_{\gamma}\right|_{A}=f_{\gamma}$ for each $\gamma \in \Gamma$ ? A little thought reveals that some additional restriction is needed. For example, let $X:=[0,1], Y:=\mathbf{R}, A:=\{0,1\}$, and $\mathcal{F}:=\left\{f_{n}\right\}_{n \in N}$, where for each $n, f_{n}$ is the uniformly continuous function defined on $A$ by $f_{n}(x):=n x$. Suppose by way of 
contradiction that $\left\{g_{n}\right\}_{n \in N} \subset C(X)$ is an equicontinuous set of extensions of the $f_{n}$. Then for each $x \in[0,1]$ we can find an interval $I_{x}:=\left(x-\delta_{x}, x+\delta_{x}\right)$ such that for any $n,\left|g_{n}(y)-g_{n}(x)\right|<1 / 2$ whenever $y \in I_{x}$. But $[0,1]$ is covered by $I_{x_{1}}, \ldots, I_{x_{k}}$ for some $x_{1}, \ldots, x_{k}$, and this implies that $n=g_{n}(1) \leq k$ for each $n$, which is clearly a contradiction.

Note that in the previous example, the set $\left\{f_{n}(1)\right\}_{n \in N}$ was unbounded. To get the right restriction, we recall that a subset $S$ of a linear topological space $Y$ is said to be bounded if for each neighborhood $V$ of $\overrightarrow{0}$ there exists $m>0$ such that $\alpha \vec{s} \in V$ for all $\vec{s} \in S$ whenever a scalar $\alpha$ satisfies $|\alpha|<m$. If we require that for each $a \in A$ the set $\left\{f_{\gamma}(a)\right\}_{\gamma \in \Gamma}$ be bounded in $Y$, then the desired extensions of the $f_{\gamma}$ exist (Theorem 8).

Before presenting the theorem, we should point out that the proof makes use of an extension defined by Dugundji in [D]. Indeed, in the process of proving the equicontinuity of $\hat{\mathcal{F}}$ on $\partial A$, we will almost repeat the proof of Dugundji's extension theorem. We also need to state a preliminary result from [D].

Recall that an open covering $\mathfrak{U}$ of a topological space $X$ is called locally finite if for each $x \in X$, there exists a neighborhood $V$ of $x$, such that $V$ meets only finitely many $U \in \mathfrak{U}$ (indeed, we may assume that $V$ is subset of each such $U$ ). If $\mathfrak{U}$ and $\mathfrak{V}$ are two open coverings of $X$, then $\mathfrak{V}$ is refinement of $\mathfrak{U}$ if for each $V \in \mathfrak{V}$ there is a set $U \in \mathfrak{U}$ containing it. We need the following result from [D].

If $(X, d)$ is a metric space, and if $A$ is a closed subset of $X$, then there exists an open covering $\mathfrak{U}$ of $X \backslash A$ such that

(i) $\mathfrak{U}$ is locally finite,

(ii) any neighborhood of any $a \in \partial A$ contains infinitely many sets from $\mathfrak{U}$,

(iii) given any neighborhood $W$ of $a \in A$, there exists a neighborhood $W^{\prime}$ of $a$ with $W^{\prime} \subset W$ such that the implication $U \cap W^{\prime} \neq \emptyset \Rightarrow U \subset W$ holds for each $U \in \mathfrak{U}$.

Using the terminology of $[D]$, we refer to such a covering as a canonical covering.

THEOREM 8. Let $(X, d)$ be a metric space, let $(Y, \varrho)$ be a locally convex metric linear space, and let $A$ be a closed subset of $X$. Suppose that $\mathcal{F}:=\left\{f_{\gamma}\right\}_{\gamma \in \Gamma}$ is an equicontinuous subset of $C(A, Y)$, 
and suppose that for each $a \in A$, the set $\left\{f_{\gamma}(a)\right\}_{\gamma \in \Gamma}$ is bounded in $Y$. Then there exists an equicontinuous subset $\hat{\mathcal{F}}:=\left\{\hat{f}_{\gamma}\right\}_{\gamma \in \Gamma}$ of $C(X, Y)$ such that $\left.\hat{f}_{\gamma}\right|_{A}=f_{\gamma}$ for each $\gamma \in \Gamma$.

Proof. Let $\mathfrak{U}$ be a canonical covering of $X \backslash A$, and define for each $U \in \mathfrak{U}$ a function $\lambda_{U}: X \backslash A \rightarrow[0,1]$ by

$$
\lambda_{U}(x):=\frac{d(x, X \backslash U)}{\sum_{U^{\prime} \in \mathfrak{U}} d\left(x, X \backslash U^{\prime}\right)} .
$$

Since $\mathfrak{U}$ is locally finite, there exists for each $x \in X \backslash A$ a neighborhood $W_{x}$ of $x$ such that $W_{x}$ meets only (say) $U_{1}, \ldots, U_{n} \in \mathfrak{U}$, and $W_{x} \subset U_{1} \cap \cdots \cap U_{n}$. Thus the sum in the denominator of (**) is finite and positive, so $\lambda_{U}$ is well-defined. Moreover, $\lambda_{U}$ is clearly continuous on $W_{x}$ (and hence at every point of $X \backslash A$ ), and for any such $x, \sum_{U \in \mathfrak{U}} \lambda_{U}(x)=\lambda_{U_{1}}(x)+\cdots+\lambda_{U_{n}}(x)=1$.

Next, for each $U$ choose $x_{U} \in U$. Then for each $x_{U}$ choose $a_{U} \in A$ such that $d\left(x_{U}, a_{U}\right)<2 d\left(x_{U}, A\right)$ (we assume of course that $a_{U}=a_{U^{\prime}}$ whenever $\left.x_{U}=x_{U^{\prime}}\right)$. We define the extensions of the $f_{\gamma}$ as follows: for each $\gamma \in \Gamma$, let

$$
\hat{f}_{\gamma}(x):= \begin{cases}\sum_{U \in \mathfrak{U}} \lambda_{U}(x) f_{\gamma}\left(a_{U}\right) & \text { if } x \notin A \\ f_{\gamma}(x) & \text { if } x \in A .\end{cases}
$$

Clearly $\hat{\mathcal{F}}:=\left\{\hat{f}_{\gamma}\right\}_{\gamma \in \Gamma}$ is equicontinuous on $\operatorname{int}(A)$, so let us first choose $x_{0} \in \partial A$ and let $\epsilon>0$. For each $\gamma$ let us write $\vec{y}_{\gamma}:=\hat{f}_{\gamma}\left(x_{0}\right)=$ $f_{\gamma}\left(x_{0}\right)$. If $B_{\varrho}\left(\vec{y}_{\gamma}, \epsilon\right)$ denotes the open ball of radius $\epsilon$ centered at $\vec{y}_{\gamma}$, we can use the translation invariance of $\varrho$ to find a convex neighborhood $V$ of $\overrightarrow{0}$ in $Y$ such that $V+\vec{y}_{\gamma}:=\left\{\vec{y}+\vec{y}_{\gamma} \mid \vec{y} \in V\right\}$ satisfies $\vec{y}_{\gamma} \in V+\vec{y}_{\gamma} \subset B_{\varrho}\left(\vec{y}_{\gamma}, \epsilon\right)$ for each $\gamma \in \Gamma$. Since there is a small ball in $V$ centered at $\overrightarrow{0}$, there is a ball of equal radius in each $V+\vec{y}_{\gamma}$ centered at $\vec{y}_{\gamma}$. Hence we may choose $\delta>0$ such that for any $\gamma, \hat{f}_{\gamma}(x)=f_{\gamma}(x) \in V+\vec{y}_{\gamma}$ whenever $x \in B_{d}\left(x_{0}, \delta\right) \cap A$. Observe that if $x_{U} \in B_{d}\left(x_{0}, \delta / 3\right)$, then

$$
\begin{aligned}
d\left(a_{U}, x_{0}\right) & \leq d\left(a_{U}, x_{U}\right)+d\left(x_{U}, x_{0}\right)<2 d\left(x_{U}, A\right)+d\left(x_{U}, x_{0}\right) \\
& \leq 2 d\left(x_{U}, x_{0}\right)+d\left(x_{U}, x_{0}\right)<2 \delta / 3+\delta / 3=\delta
\end{aligned}
$$

Hence for any $\gamma \in \Gamma, x_{U} \in B_{d}\left(x_{0}, \delta / 3\right) \Rightarrow f_{\gamma}\left(a_{U}\right) \in V+\vec{y}_{\gamma}$. 
Finally, using the fact that $\mathfrak{U}$ is canonical, choose a neighborhood $W_{0}$ of $x_{0}$, with $W_{0} \subset B_{d}\left(x_{0}, \delta / 3\right)$,such that $U \cap W_{0} \neq \emptyset \Rightarrow U \subset$ $B_{d}\left(x_{0}, \delta / 3\right)$. Then $x \in W_{0} \backslash A$ implies that $x$ belongs only to some $U_{1}, \ldots, U_{n}$ and $x_{U_{1}}, \ldots, x_{U_{n}} \in B_{d}\left(x_{0}, \delta / 3\right)$. Hence for any $\gamma, \hat{f}_{\gamma}(x)$ is a convex combination

$$
\hat{f}_{\gamma}(x)=\lambda_{U_{1}}(x) f_{\gamma}\left(a_{U_{1}}\right)+\cdots+\lambda_{U_{n}}(x) f_{\gamma}\left(a_{U_{n}}\right)
$$

of vectors $f_{\gamma}\left(a_{U_{i}}\right)$ belonging to the convex set $V+\vec{y}_{\gamma}$. Therefore $\hat{f}_{\gamma}(x) \in V+\vec{y}_{\gamma}$, which implies $\hat{f}_{\gamma}(x) \in B_{\varrho}\left(\vec{y}_{\gamma}, \epsilon\right)$. This proves that $\hat{\mathcal{F}}$ is equicontinuous at each $x \in \partial A$.

To finish the proof, let $x_{0} \in X \backslash A$ and let $\epsilon>0$. As before, let us write for each $\gamma, \vec{y}_{\gamma}:=\hat{f}_{\gamma}\left(x_{0}\right)=\sum_{i=1}^{n} \lambda_{U_{i}}\left(x_{0}\right) f_{\gamma}\left(a_{U_{i}}\right)$, where $U_{1}, \ldots, U_{n}$ are precisely those $U \in \mathfrak{U}$ which contain $x_{0}$, and let $W_{0} \subset$ $U_{1} \cap \cdots \cap U_{n}$ be a neighborhood of $x_{0}$ disjoint from every other $U$. Again let $V$ be a convex neighborhood of $\overrightarrow{0}$ satisfying $\vec{y}_{\gamma} \in V+\vec{y}_{\gamma} \subset$ $B_{\varrho}\left(\vec{y}_{\gamma}, \epsilon\right)$ for each $\gamma \in \Gamma$. With $n$ fixed, it is known that there exists a neighborhood $V^{\prime}$ of $\overrightarrow{0}$ contained in $V$ such that $\vec{v}_{1}+\cdots+\vec{v}_{n} \in V$ for any $n$ vectors $\vec{v}_{1}, \ldots, \vec{v}_{n} \in V^{\prime}$. Now since each set $\left\{f_{\gamma}\left(a_{U_{i}}\right)\right\}_{\gamma \in \Gamma}$ is bounded, so is the union of these $n$ sets. Hence there exists a number $m>0$ such that for any $\gamma, \alpha f_{\gamma}\left(a_{U_{1}}\right), \ldots, \alpha f_{\gamma}\left(a_{U_{n}}\right) \in V^{\prime}$ for any $\alpha$ with $|\alpha|<m$. Then since $\lambda_{1}, \ldots, \lambda_{n}$ are continuous, we can find a sufficiently small neighborhood $W_{0}^{\prime} \subset W_{0}$ of $x_{0}$ such that whenever $x \in W_{0}^{\prime}$, we have $\left(\lambda_{U_{i}}(x)-\lambda_{U_{i}}\left(x_{0}\right)\right) f_{\gamma}\left(a_{U_{i}}\right) \in V^{\prime}$ for $1 \leq i \leq n$ and for every $\gamma \in \Gamma$. Hence the vector

$$
\begin{aligned}
\hat{f}_{\gamma}(x)-\vec{y}_{\gamma}= & \left(\lambda_{U_{1}}(x)-\lambda_{U_{1}}\left(x_{0}\right)\right) f_{\gamma}\left(a_{U_{1}}\right) \\
& +\cdots+\left(\lambda_{U_{n}}(x)-\lambda_{U_{n}}\left(x_{0}\right)\right) f_{\gamma}\left(a_{U_{n}}\right)
\end{aligned}
$$

belongs to $V$, so $\hat{f}_{\gamma}(x) \in V+\vec{y}_{\gamma} \Rightarrow \hat{f}_{\gamma}(x) \in B_{\varrho}\left(\vec{y}_{\gamma}, \epsilon\right)$ whenever $x \in W_{0}^{\prime}$. Since the choice of $W_{0}^{\prime}$ was independent of $\gamma$, this proves that $\hat{\mathcal{F}}$ is equicontinuous at $x_{0}$.

REMARK. It is clear that we cannot remove the requirement in the above theorem that $A$ be closed. For example, the set $\left\{f_{n}(x):=\right.$ $\left.x^{n}\right\}_{n \in N}$ of real-valued functions is equicontinuous on the interval $[0,1)$, but not on $[0,1]$.

The following corollary is a slight variation of Theorem 8, and in view of the Ascoli-Arzela theorem, it takes on a pleasing form. 
COROllary 9. Let $(X, d)$ be a locally compact metric space, let $(Y, \varrho)$ be a locally convex metric linear space, and let $A \subset X$ be closed. Let us consider the compact-open topology on $C(A, Y)$ and $C(X, Y)$, and suppose that $\mathcal{F}:=\left\{f_{\gamma}\right\}_{\gamma \in \Gamma}$ is a compact subset of $C(A, Y)$ for some indexing set $\Gamma$. Then there exists a compact subset $\hat{\mathcal{F}}:=\left\{\hat{f}_{\gamma}\right\}_{\gamma \in \Gamma}$ of $C(X, Y)$ such that $\left.\hat{f}_{\gamma}\right|_{A}=f_{\gamma}$ for each $\gamma \in \Gamma$.

Proof. We will show that the set $\hat{\mathcal{F}}$ as defined in Theorem 8 is the desired subset of $C(X, Y)$. We already know that $\hat{\mathcal{F}}$ is an equicontinuous set of extensions of the $f_{\gamma}$. By the Ascoli-Arzela theorem, we can show that $\hat{\mathcal{F}}$ has compact closure by proving that for each $x_{0} \in X \backslash A$, the set $\left\{\hat{f}_{\gamma}\left(x_{0}\right)\right\}_{\gamma \in \Gamma}$ is compact in $Y$ (clearly this true for any $\left.x_{0} \in A\right)$. To prove this, let $x_{0} \in X \backslash A$ and let $\left\{\hat{f}_{k}\left(x_{0}\right)\right\}_{k=1}^{\infty}$ be any sequence (we assume that $0,1,2, \ldots \in \Gamma$ ). Since $\mathcal{F}$ is compact, the sequence $\left\{f_{k}\right\}_{k=1}^{\infty}$ has a convergent subsequence $\left\{f_{k_{m}}\right\}_{m=1}^{\infty}$ such that $f_{k_{m}} \stackrel{m}{\rightarrow} f_{0}{ }^{2}$ for some $f_{0} \in \mathcal{F}$. We will prove the compactness of $\left\{\hat{f}_{\gamma}\left(x_{0}\right)\right\}_{\gamma \in \Gamma}$ by reindexing the convergent subsequence as $\left\{f_{m}\right\}_{m=1}^{\infty}$ and showing that $\hat{f}_{m}\left(x_{0}\right) \rightarrow \hat{f}_{0}\left(x_{0}\right)$.

As in the proof of Theorem 7 , let $U_{1}, \ldots, U_{n}$ be precisely those $U \in \mathfrak{U}$ which contain $x_{0}$ and let $a_{U_{1}}, \ldots, a_{U_{n}} \in A$ be the corresponding points in $A$. Since convergence in the compact-open topology on $C(A, Y)$ implies pointwise convergence on $A$, we have

$$
f_{m}\left(a_{U_{1}}\right) \stackrel{m}{\rightarrow} f_{0}\left(a_{U_{1}}\right), \ldots, f_{m}\left(a_{U_{n}}\right) \stackrel{m}{\rightarrow} f_{0}\left(a_{U_{n}}\right)
$$

in $Y$. Since scalar multiplication is continuous, we also have

$$
\begin{aligned}
\lambda_{U_{1}}\left(x_{0}\right) f_{m}\left(a_{U_{1}}\right) & \stackrel{m}{\rightarrow} \lambda_{U_{1}}\left(x_{0}\right) f_{0}\left(a_{U_{1}}\right), \ldots, \lambda_{U_{n}}\left(x_{0}\right) f_{m}\left(a_{U_{n}}\right) \\
& \stackrel{m}{\rightarrow} \lambda_{U_{n}}\left(x_{0}\right) f_{0}\left(a_{U_{n}}\right),
\end{aligned}
$$

and since vector addition is also continuous,

$$
\sum_{i=1}^{n} \lambda_{U_{i}}\left(x_{0}\right) f_{m}\left(a_{U_{i}}\right) \stackrel{m}{\rightarrow} \sum_{i=1}^{n} \lambda_{U_{i}}\left(x_{0}\right) f_{0}\left(a_{U_{i}}\right),
$$

which is equivalent to $\hat{f}_{m}\left(x_{0}\right) \rightarrow \hat{f}_{0}\left(x_{0}\right)$.

We will finish the proof by showing that $\hat{\mathcal{F}}$ is in fact closed. Let $\left\{\hat{f}_{\delta}\right\}_{\delta \in \Delta}$ be any net in $\hat{\mathcal{F}}$ converging to a function in $C(X, Y)$, where

${ }^{2}$ For brevity, we will hereafter use notation of the form $P_{i} \stackrel{i}{\rightarrow} Q$ to indicate that $P_{i} \rightarrow Q$ as $i \rightarrow \infty$. 
$\Delta$ is a directed set. Since $\mathcal{F}$ is closed, we have $f_{\delta} \rightarrow f_{0}$ for some $f_{0} \in \mathcal{F}$. We are done if we show that $\hat{f}_{\delta} \rightarrow \hat{f}_{0}$, and this can be proved by showing that for any $x_{0} \in X \backslash A$, we have $\hat{f}_{\delta}\left(x_{0}\right) \rightarrow \hat{f}_{0}\left(x_{0}\right)$ in $Y$, because the compact-open topology and the pointwise convergence topology coincide on $\hat{\mathcal{F}}$. Having fixed $x_{0}$, let $U_{1}, \ldots, U_{n}$ and $a_{U_{1}}, \ldots, a_{U_{n}} \in A$ be as described above. By an argument similar to that used above, we have the following three results:

$$
\begin{aligned}
& f_{\delta}\left(a_{U_{1}}\right) \stackrel{\delta}{\rightarrow} f_{0}\left(a_{U_{1}}\right), \ldots, f_{\delta}\left(a_{U_{n}}\right) \stackrel{\delta}{\rightarrow} f_{0}\left(a_{U_{n}}\right), \\
& \lambda_{U_{1}}\left(x_{0}\right) f_{\delta}\left(a_{U_{1}}\right) \stackrel{\delta}{\rightarrow} \lambda_{U_{1}}\left(x_{0}\right) f_{0}\left(a_{U_{1}}\right), \ldots, \lambda_{U_{n}}\left(x_{0}\right) f_{\delta}\left(a_{U_{n}}\right) \\
& \stackrel{\delta}{\rightarrow} \lambda_{U_{n}}\left(x_{0}\right) f_{0}\left(a_{U_{n}}\right) \\
& \sum_{i=1}^{n} \lambda_{U_{i}}\left(x_{0}\right) f_{\delta}\left(a_{U_{i}}\right) \stackrel{\delta}{\rightarrow} \sum_{i=1}^{n} \lambda_{U_{i}}\left(x_{0}\right) f_{0}\left(a_{U_{i}}\right),
\end{aligned}
$$

the last result being equivalent to $\hat{f}_{\delta}\left(x_{0}\right) \rightarrow \hat{f}_{0}\left(x_{0}\right)$.

The author is indebted to Professor Yuri Abramovich for his advice and encouragement.

\section{REFERENCES}

[D] J. Dugundji, An extension of Tietze's theorem, Pacific J. Math., 1 (1951), 353-367.

[GJ] L. Gillman and M. Jerison, Rings of Continuous Functions, Princeton, N.J. 1960.

[H] F. Hausdorff, Über halbstetige Funktionen und deren Verallgemeinerung, Math. Z., 5 (1919), 292-309.

[K] M. Katětov, On real-valued functions in topological spaces, Fund. Math., 38 (1951), 85-91; Correction, 40 (1953), 203-205.

[Ke] B. Kerékjártó, Vorlesungen über Topologie, Berlin 1923.

[M] M. Mandelkern, On the uniform continuity of Tietze extensions, Arch. Math., 55 (1990), 387-388.

[T] H. Tietze, Über Functionen, die auf einer abgeschlossenen Menge stetig sind, J. Reine Angew. Math., 145 (1915), 9-14.

[U] P. Urysohn, Über die Mächtigkeit der zusammenhängenden Mengen, Math. Ann., 94 (1925), 262-295.

Received August 31, 1992 and in revised form April 27, 1993. 



\title{
PACIFIC JOURNAL OF MATHEMATICS
}

Founded by E. F. Beckenbach (1906-1982) and F. Wolf (1904-1989)

\section{EDITORS}

Sun-Yung Alice Chang (Managing Editor)

University of California

Los Angeles, CA 90095-1555

pacific@math.ucla.edu

F. Michael Christ

University of California

Los Angeles, CA 90095-1555

christ@math.ucla.edu

Thomas Enright

University of California

San Diego, La Jolla, CA 92093

tenright@ucsd.edu

Nicholas Ercolani

University of Arizona

Tucson, AZ 85721

ercolani@math.arizona.edu
Robert Finn

Stanford University

Stanford, CA 94305

finn@gauss.stanford.edu

Vaughan F. R. Jones

University of California

Berkeley, CA 94720

vfr@math.berkeley.edu

Steven Kerckhoff

Stanford University

Stanford, CA 94305

spk@gauss.stanford.edu
Martin Scharlemann

University of California

Santa Barbara, CA 93106

mgscharl@math.ucsb.edu

\section{Gang Tian}

Courant Institute

New York University

New York, NY 10012-1100

tiang@taotao.cims.nyu.edu

V. S. Varadarajan

University of California

Los Angeles, CA 90095-1555

vsv@math.ucla.edu

\section{SUPPORTING INSTITUTIONS}

\section{CALIFORNIA INSTITUTE OF TECHNOLOGY \\ NEW MEXICO STATE UNIVERSITY \\ OREGON STATE UNIVERSITY \\ STANFORD UNIVERSITY \\ UNIVERSITY OF ARIZONA \\ UNIVERSITY OF BRITISH COLUMBIA \\ UNIVERSITY OF CALIFORNIA UNIVERSITY OF HAWAII}

\author{
UNIVERSITY OF MONTANA \\ UNIVERSITY OF NEVADA, RENO \\ UNIVERSITY OF OREGON \\ UNIVERSITY OF SOUTHERN CALIFORNIA \\ UNIVERSITY OF UTAH \\ UNIVERSITY OF WASHINGTON \\ WASHINGTON STATE UNIVERSITY
}

The supporting Institutions listed above contribute to the cost of publication of this Journal, but they are not owners or publishers and have no responsibility for its contents or policies.

Manuscripts must be prepared in accordance with the instructions provided on the inside back cover.

The Pacific Journal of Mathematics (ISSN 0030-8730) is published monthly except for July and August. Regular subscription rate: $\$ 215.00$ a year (10 issues). Special rate: $\$ 108.00$ a year to individual members of supporting institutions.

Subscriptions, orders for back issues published within the last three years, and changes of subscribers address should be sent to Pacific Journal of Mathematics, P.O. Box 4163, Berkeley, CA 94704-0163, U.S.A. Prior back issues are obtainable from Kraus Periodicals Co., Route 100, Millwood, NY 10546.

The Pacific Journal of Mathematics at the University of California, c/o Department of Mathematics, 981 Evans Hall, Berkeley, CA 94720 (ISSN 0030-8730) is published monthly except for July and August. Second-class postage paid at Berkeley, CA 94704, and additional mailing offices. POSTMASTER: send address changes to Pacific Journal of Mathematics, P.O. Box 6143, Berkeley, CA 94704-0163.

\author{
PUBLISHED BY PACIFIC JOURNAL OF MATHEMATICS at University of California, \\ Berkeley, CA 94720, A NON-PROFIT CORPORATION \\ This publication was typeset using AMS-LATEX, \\ the American Mathematical Society's TEX macro system. \\ Copyright (C) 1995 by Pacific Journal of Mathematics
}




\section{PACIFIC JOURNAL OF MATHEMATICS}

Volume 169 No. $1 \quad$ May 1995

Minimal sets of periods for torus maps via Nielsen numbers

Lluís AlsedÀ, StewART BALdWin, JAUME Llibre,

RICHARD SWANSON and WIESLAW SZLENK

Diagonalizing Hilbert cusp forms

Timothy ATWILL

A splitting criterion for rank 2 vector bundles on $\mathbf{P}^{n}$

EDOARDO BALLICO

Controlling Tietze-Urysohn extensions

MARC FRANTZ

Length of Julia curves

DAVID H. HAMILTON

On the uniqueness of capillary surfaces over an infinite strip

JENN-FANG HWANG

Volume estimates for log-concave densities with application to iterated 107 convolutions

MARIUS JUNGE

A reflection principle in complex space for a class of hypersurfaces and mappings

FRANCINE ANTOINETTE MEYLAN

Jean Bourgain's analytic partition of unity via holomorphic martingales 161

PAUL F.X. MÜLLER

Characters of Brauer's centralizer algebras 